\title{
Diophantine classes, dimension and Denjoy maps
}

\author{
by
}

Bryna Kra (University Park, PA) and Jörg Schmeling (Lund)

\section{Introduction}

1.1. History. Poincaré [13] showed that to each orientation preserving homeomorphism $f$ of the circle $S^{1}=\mathbb{R} / \mathbb{Z}$ is associated a unique real parameter $\alpha \in[0,1)$, called the rotation number, so that the orbit structure of $f$ is the same as that of the rigid rotation $R_{\alpha}$, where $R_{\alpha}(t)=(t+\alpha) \bmod 1$, provided that $\alpha$ is irrational. More precisely, Poincaré showed that $f$ and $R_{\alpha}$ are semi-conjugate, meaning there exists an orientation preserving self-mapping $h$ of the circle so that $h \circ f=R_{\alpha} \circ h$.

We say that $f$ is minimal if for each $x \in S^{1}$, the orbit under $f$ is dense. (In this setup, density of the orbit for a single $x \in S^{1}$ is equivalent to density for all $x$.) More generally, for a homeomorphism $f$ the set $\Omega$ is minimal if $\Omega$ is non-empty, compact, invariant and minimal with respect to these properties. This is equivalent to saying that $\Omega$ is non-empty, $f(\Omega)=\Omega$ and each forward $f$-orbit in $\Omega$ is dense in $\Omega$. It follows from Poincaré's Theorem that if $f$ is minimal, then $f$ is conjugate to the rotation by $\alpha$. That is, there exists an orientation preserving homeomorphism of the circle $h$ so that $f=h^{-1} \circ R_{\alpha} \circ h$. We assume throughout that all rotation numbers are irrational, even when not explicitly stated.

Half a century later, Denjoy [3] showed that if the derivative of $f$ is of bounded variation, then $f$ is minimal and so conjugate to a rotation. Denjoy also constructed examples of $C^{1}$ diffeomorphisms that are not conjugate to rotations. Herman [6] constructed $C^{1+\delta}$ maps that are not conjugate to rotations for any $0<\delta<1$. In these examples, the minimal set of $f$ is necessarily a Cantor set $\Omega$ and is the set of accumulation points of the forward $f$ orbit of every point $t \in S^{1}$. We refer to any orientation preserving map that is not conjugate to a rotation as a Denjoy map. $28 \mathrm{C} 99$.

2000 Mathematics Subject Classification: Primary 58F11, 58F12; Secondary 28D99,

Key words and phrases: Denjoy example, Hausdorff dimension, Diophantine classes.

Jörg Schmeling was supported by the Leopoldina-Förderpreis Foundation. 
1.2. Summary of results. The rotation number has a strong effect on properties of the diffeomorphism. One area that has been well understood is the relation between the differentiability of $f$, the differentiability of the conjugation and the arithmetic properties of the rotation number. (See, for example, Herman [6], Yoccoz [14] and Katznelson and Ornstein [8].) Without stating any precise theorem, we note that the results differ sharply for Diophantine and for Liouville rotation numbers.

Here we study the effect of the rotation number on a $C^{1+\delta}$ Denjoy example. In particular, we consider the relation between the arithmetic properties of the rotation number and the Hausdorff dimension of the minimal set $\Omega$ of a $C^{1+\delta}$ example. See Section 2 for the definitions.

We prove:

Theorem 1.1. Assume that $0<\delta<1$ and that $\alpha \in(0,1)$ is of Diophantine class $\nu \in(0, \infty)$. Then an orientation preserving $C^{1+\delta}$ diffeomorphism of the circle with rotation number $\alpha$ and minimal set $\Omega_{\alpha}^{\delta}$ satisfies

$$
\operatorname{dim}_{\mathrm{B}} \Omega_{\alpha}^{\delta} \geq \delta \quad \text { and } \quad \operatorname{dim}_{\mathrm{H}} \Omega_{\alpha}^{\delta} \geq \delta / \nu .
$$

Furthermore, these results are sharp.

Norton [11] proved a preliminary result along these lines, showing that the upper box dimension of the minimal set of a $C^{1+\delta}$ Denjoy map is bounded from below by $\delta$. Katznelson and Ornstein [9] have shown that for some specific Denjoy maps, the Hausdorff dimension of the minimal set depends on the differentiability. Pinto [12] proved the special case when the rotation number is the golden mean. Our results include these and show that while the box dimension does not depend on the rotation number of the Denjoy example, the more subtle Hausdorff dimension does.

The estimates on dimension give a stronger version of Denjoy's original theorem about conjugacy that takes the arithmetic of the rotation number into account. Namely, we have the following immediate corollary to Theorem 1.1:

Corollary 1.2. Assume that $f$ is an orientation preserving homeomorphism of the circle with rotation number $\alpha \in(0,1)$ and that $\alpha$ is of Diophantine class $\nu \in(0, \infty)$. Let $\Omega$ denote the minimal set of $f$. If $\operatorname{dim} \Omega=$ $\delta / \nu \in(0,1)$, then $f \notin C^{1+\beta}$ for any $\beta>\delta$.

\section{Preliminaries}

2.1. Construction of Denjoy maps. We review the construction of the classic Denjoy map. (See [7] for details of the construction.) The classic Denjoy map is used to show that the estimates in Theorem 1.1 are sharp. 
Definition 2.1. A Denjoy sequence $D^{\delta}$ of class $\delta$ is an infinite collection $\left\{\ell_{n}\right\}_{n \in \mathbb{Z}}$ of lengths (non-negative numbers) so that

$$
\sum_{n \in \mathbb{Z}} \ell_{n} \leq 1
$$

and

$$
\varliminf_{n \rightarrow \pm \infty} \frac{\log \left|\ell_{n}-\ell_{n+1}\right|}{\log \ell_{n}}=1+\delta .
$$

Definition 2.2. The classic Denjoy sequence, denoted by $D_{0}^{\delta}$, consists of the lengths

$$
\ell_{n}=c_{\delta}(|n|+1)^{-1 / \delta},
$$

where $c_{\delta}^{-1}=\sum_{n \in \mathbb{Z}}(|n|+1)^{-1 / \delta}$.

We denote the set of all Denjoy sequences of class $\delta$ by $\mathcal{D}^{\delta}$.

Let $\alpha \in(0,1)$ be irrational and let $\delta \in(0,1)$. Let $D^{\delta}$ be a Denjoy sequence of class $\delta$. In order to obtain a Denjoy map, we "blow up" each point $n \alpha$ of the orbit of 0 under the rotation by $\alpha$ to an open interval $J_{n}$ of length $\ell_{n} \in D^{\delta}$. At each step we rescale the remaining part of the circle, maintaining the total length equal to 1 .

A Denjoy map $f$ is defined by mapping the interval $J_{n}$ diffeomorphically to $J_{n+1}$ with derivative of absolute value 1 at the endpoints. By continuity, $f$ is defined on the entire circle and by choice of the sequence $\ell_{n}, f$ can be chosen to be $C^{1+\delta}$. By placement of the intervals $J_{n}$ in the same order as the rotation by $\alpha$, the resulting $f$ has rotation number $\alpha$.

We set

$$
\Omega_{\alpha}^{\delta}=\Omega_{\alpha}^{\delta}\left(D^{\delta}\right)=S^{1} \backslash \bigcup_{n \in \mathbb{Z}} J_{n}
$$

For $x, y \in S^{1}$, we let $(x, y)$ denote the shorter of the two arcs on the circle determined by $x$ and $y$. (If this interval is not unique, we take the interval in the positive orientation.) For real $x$, let $\|x\|$ denote the distance to the nearest integer. For an interval $J \subset S^{1},|J|$ denotes the length of the interval.

Let $h: \Omega_{\alpha}^{\delta} \rightarrow S^{1}$ be the semi-conjugacy determined by Poincaré's Theorem. For $x, y$ not in the orbit of $\alpha$ (meaning $x, y \in S^{1} \backslash\{n \alpha: n \in \mathbb{Z}\}$ ), $h^{-1}$ is well defined and we have

$$
d_{\Omega_{\alpha}^{\delta}}\left(h^{-1}(x), h^{-1}(y)\right)=\sum_{\{n: n \alpha \in(x, y)\}}\left|J_{n}\right|+\left(1-\sum_{n \in \mathbb{Z}}\left|J_{n}\right|\right)\|x-y\| .
$$

If $x \in\{n \alpha: n \in \mathbb{Z}\}$ (similarly for $y$ ), $h^{-1}(x)$ consists of two points and we choose the appropriate preimage. For example, if the interval $(x, y)$ is positively oriented for $h^{-1}(x)$ we take the right preimage (equivalently, the point closer to $\left.h^{-1}(y)\right)$. 
We note that the total length $\sum \ell_{n}$ may be strictly less than 1 . In this case, the invariant measure associated to $\Omega_{\alpha}^{\delta}$ has positive Lebesgue measure and the dimension properties are not relevant.

We also have some preliminary estimates on sizes of complementary intervals for general Denjoy maps, and not just for the classic Denjoy map. These bounds are summarized in the following lemma:

Lemma 2.3. If $D^{\delta} \in \mathcal{D}^{\delta}$, then for all $0<\theta<\delta<1$ there exists $n_{\theta}$ such that for all $n>n_{\theta}$,

$$
\left|J_{n}\right|>n^{-1 / \theta} \text {. }
$$

Proof. We note that if $f$ is a $C^{1+\delta}$ diffeomorphism then $f^{-1}$ is also a $C^{1+\delta}$ diffeomorphism.

Assume that $D^{\delta} \in \mathcal{D}^{\delta}$. Then for $0<\theta<\delta<1$ there exists $n_{\theta}$ such that for all $n \geq n_{\theta}$,

$\left|\frac{\left|J_{n}\right|}{\left|J_{n+1}\right|}-D_{h^{-1}((n+1) \alpha)} f^{-1}\right| \leq\left|\max _{x \in J_{n+1}} D_{x} f^{-1}-D_{h^{-1}((n+1) \alpha)} f^{-1}\right| \leq\left|J_{n+1}\right|^{\theta}$.

Rewriting, we have

$$
\left|J_{n}\right| \leq\left|J_{n+1}\right|\left(D_{h^{-1}((n+1) \alpha)} f^{-1}+\left|J_{n+1}\right|^{\theta}\right) .
$$

Setting

$$
a_{n}=\left|J_{n}\right| n^{\beta},
$$

where $\beta=\theta^{-1}>1$, it suffices to show that for $n$ sufficiently large, $a_{n}$ is bounded away from 0 . Inequality (2) becomes

$$
\frac{a_{n}}{n^{\beta}} \leq \frac{a_{n+1}}{(n+1)^{\beta}}\left(1+\frac{a_{n+1}^{\theta}}{n+1}\right) .
$$

Equivalently, for $n>n_{\theta}$ and bounded $a_{n+1}$ we have

$$
\frac{a_{n}}{a_{n+1}} \leq\left(1+\frac{a_{n+1}^{\theta}}{n+1}\right) \cdot\left(\frac{n}{n+1}\right)^{\beta} \leq \exp \left(\frac{a_{n+1}^{\theta}-\beta}{n+1}\right) .
$$

For $a_{n+1}^{\theta}<\beta$ the right side of inequality (3) is less than 1, again for sufficiently large $n$. Hence $a_{n}$ is bounded away from 0 .

2.2. Dimension. Let $Y$ be a bounded subset of a compact metric space $X$. Let $N(\varepsilon)$ denote the minimal number of $\varepsilon$ balls needed to cover $Y$.

Definition 2.4. For a subset $Y$ of a compact metric space $X$, the upper box dimension of $Y$, denoted by $\operatorname{dim}_{\mathrm{UB}} Y$ is given by

$$
\varlimsup_{\varepsilon \rightarrow 0} \frac{\log N(\varepsilon)}{\log (1 / \varepsilon)} .
$$

The lower box dimension $\operatorname{dim}_{\mathrm{LB}}$ is defined similarly, with the $\overline{\lim }$ replaced by $\underline{\text { lim. }}$. 
If $\operatorname{dim}_{\mathrm{UB}} Y$ and $\operatorname{dim}_{\mathrm{LB}} Y$ both exist and are equal, we define the box dimension of $Y$ to be this value, and write $\operatorname{dim}_{\mathrm{B}} Y=\operatorname{dim}_{\mathrm{UB}} Y=\operatorname{dim}_{\mathrm{LB}} Y$.

For a subset $U$ of a metric space, we let $\operatorname{diam}(U)$ denote the diameter of the set $U$.

Definition 2.5. Let $s \in[0, \infty]$. The $s$-dimensional Hausdorff measure $\mathcal{H}^{s}(Y)$ of a subset $Y$ of a metric space $X$ is defined by

$\mathcal{H}^{s}(Y)=\lim _{\varepsilon \rightarrow 0} \inf \left\{\sum_{i=1}^{\infty}\left(\operatorname{diam}\left(U_{i}\right)\right)^{s}: Y \subset \bigcup_{i=1}^{\infty} U_{i}\right.$ and $\left.\sup _{i=1, \ldots, \infty} \operatorname{diam}\left(U_{i}\right) \leq \varepsilon\right\}$.

It is easy to see that there exists a unique $s_{0}=s_{0}(Y)$ such that

$$
\mathcal{H}^{s}(Y)= \begin{cases}\infty & \text { for } s<s_{0} \\ 0 & \text { for } s>s_{0}\end{cases}
$$

Definition 2.6. The unique number $s_{0}$ given by (4) is defined to be the Hausdorff dimension of $Y$ and is denoted by $\operatorname{dim}_{\mathrm{H}} Y$.

Standard arguments show that for a subset $Y$ of a metric space $X$,

$$
\operatorname{dim}_{\mathrm{H}} Y \leq \operatorname{dim}_{\mathrm{LB}} Y \leq \operatorname{dim}_{\mathrm{UB}} Y
$$

and that these inequalities may be strict.

The box dimension can also be defined in terms of covering sums, as in the case of the Hausdorff dimension, with the only change being that the covering intervals all have equal length. We note that in order to estimate the box dimension, it suffices that the lengths of the covering intervals tend to 0 along a geometric sequence.

Lastly, we define the Hausdorff dimension of a measure:

Definition 2.7. Let $\mu$ be a Borel probability measure on $X$. Then the Hausdorff dimension of the measure $\mu$ is defined by

$$
\operatorname{dim}_{\mathrm{H}} \mu=\inf _{Y}\left\{\operatorname{dim}_{\mathrm{H}} Y: \mu(Y)=1\right\} .
$$

A survey of the methods and results in dimension theory can be found in [4].

2.3. Diophantine classes. Discriminating the Denjoy sets necessitates the following number-theoretic definitions. See Cassels [2] for an overview of Diophantine classes.

Definition 2.8. An irrational number $\alpha$ is of Diophantine class $\nu=$ $\nu(\alpha) \in \mathbb{R}^{+}$if

$$
\|q \alpha\|<1 / q^{\mu}
$$

has infinitely many solutions in integers $q$ for $\mu<\nu$ and at most finitely many for $\mu>\nu$. 
If $\alpha$ is not of any Diophantine class $\nu \in \mathbb{R}^{+}$then $\alpha$ is said to be a Liouville number.

If $\alpha$ is of Diophantine class $\nu$, we write $\alpha \in \operatorname{Dioph}(\nu)$. In a slight abuse of terminology, we say that a Liouville number $\alpha \in(0,1)$ has infinite Diophantine class and write $\alpha \in \operatorname{Dioph}(\infty)$.

We denote by $\left[a_{1}, a_{2}, \ldots\right]$ the (standard) continued fraction expansion of $\alpha$. We use $\left\{q_{n}\right\}$ to denote the sequence of denominators of the corresponding finite approximants. Then

$$
\frac{1}{q_{n}\left(a_{n}+2\right)} \leq\left\|q_{n} \alpha\right\| \leq \frac{1}{a_{n} q_{n}}
$$

and

$$
q_{n+1}=a_{n} q_{n}+q_{n-1} .
$$

3. Statement of results. We now have the notation for stating our results in detail.

Theorem 3.1. Let $0<\delta<1$. Assume that the irrational $\alpha \in(0,1)$ and that $\left\{q_{n}\right\}$ is the sequence of the denominators for the continued fraction convergents. Then

$$
\min _{D^{\delta} \in \mathcal{D}^{\delta}} \operatorname{dim}_{\mathrm{H}} \Omega_{\alpha}^{\delta}\left(D^{\delta}\right)=\delta \varliminf_{n \rightarrow \infty} \frac{\log q_{n}}{\log q_{n+1}} .
$$

Furthermore, the minimum dimension is attained for the classic Denjoy sequence $D_{0}^{\delta}$ of class $\delta$.

We postpone the proof of Theorem 3.1 until Section 5, and instead state and prove several corollaries of the theorem.

Corollary 3.2. Let $0<\delta<1$. If $\alpha \in(0,1)$ is of Diophantine class $\nu \in \mathbb{R}^{+}$, then

$$
\min _{D^{\delta} \in \mathcal{D}^{\delta}} \operatorname{dim}_{\mathrm{H}} \Omega_{\alpha}^{\delta}\left(D^{\delta}\right)=\delta / \nu \quad \text { and } \quad \operatorname{dim}_{\mathrm{H}} \Omega_{\alpha}^{\delta}\left(D_{0}^{\delta}\right)=\delta / \nu .
$$

Proof. If $\alpha$ is of Diophantine class $\nu$, then

$$
\begin{aligned}
\underline{\varliminf_{n \rightarrow \infty} \frac{\log q_{n}}{\log q_{n+1}}} & =\varliminf_{n \rightarrow \infty} \frac{\log q_{n}}{\log \left(a_{n} q_{n}+q_{n-1}\right)} \\
& =\varliminf_{n \rightarrow \infty} \frac{\log q_{n}}{\log \left(a_{n} q_{n}\right)} \cdot \frac{\log \left(a_{n} q_{n}\right)}{\log \left(a_{n} q_{n}+q_{n-1}\right)} \\
& =\varliminf_{n \rightarrow \infty} \frac{\log q_{n}}{\log q_{n}^{\nu}}=\frac{1}{\nu} .
\end{aligned}
$$

Hence by Theorem $3.1 \min _{D^{\delta} \in \mathcal{D}^{\delta}} \operatorname{dim}_{\mathrm{H}} \Omega_{\alpha}^{\delta}\left(D^{\delta}\right)=\delta / \nu$. 
Corollary 3.2 proves the statements of Theorem 1.1 on Hausdorff dimension. The remaining part of Theorem 1.1, concerning box dimension, is proved in Section 4.

Corollary 3.3. Let $0<\delta<1$ and let $\alpha \in(0,1)$ be irrational. Then

$$
\min _{D^{\delta} \in \mathcal{D}^{\delta}} \operatorname{dim}_{\mathrm{H}} \Omega_{\alpha}^{\delta}\left(D^{\delta}\right)=\delta \quad \text { and } \quad \operatorname{dim}_{\mathrm{H}} \Omega_{\alpha}^{\delta}\left(D_{0}^{\delta}\right)=\delta
$$

for Lebesgue almost all $\alpha$.

Proof. This follows immediately from Corollary 3.2, since almost every number (with respect to Lebesgue measure) is of Diophantine class $1+\varepsilon$ for any $\varepsilon>0$.

Another immediate consequence of Corollary 3.2 is the following:

Corollary 3.4. Let $0<\delta<1$. If $\alpha \in(0,1)$ is a Liouville number then

$$
\min _{D^{\delta} \in \mathcal{D}^{\delta}} \operatorname{dim}_{\mathrm{H}} \Omega_{\alpha}^{\delta}\left(D^{\delta}\right)=0 .
$$

4. The box dimension of $\Omega_{\alpha}^{\delta}\left(D_{0}^{\delta}\right)$. We start by computing the box dimension for the classic map.

For simplicity, we do not give the optimal result in the following estimate, as the constants do not affect our results. We use $[x]$ to denote the integer part of $x$.

Lemma 4.1. Let $\left\{a_{i}\right\}$ be a sequence of positive numbers and let $n \in \mathbb{N}$. Then

$$
\sum_{k=1}^{n}\left(\left[\frac{n a_{k}}{\sum_{i=1}^{n} a_{i}}\right]+1\right) \leq 3 n .
$$

Proof. Without loss of generality, we assume that $a_{1} \leq \ldots \leq a_{n}$. Let

$$
m=\left|\left\{1 \leq i \leq n: a_{i}<\sum_{k=1}^{n} a_{k} / n\right\}\right| .
$$

Clearly $m<n$. Then

$$
\begin{aligned}
\sum_{k=1}^{n}\left(\left[\frac{n a_{k}}{\sum_{i=1}^{n} a_{i}}\right]+1\right) & \leq m+\sum_{k=m+1}^{n}\left(\left[\frac{n a_{k}}{\sum_{i=1}^{n} a_{i}}\right]+1\right) \\
& \leq m+2 \sum_{k=m+1}^{n} \frac{n a_{k}}{\sum_{i=1}^{n} a_{i}} \leq m+2 n \leq 3 n .
\end{aligned}
$$

Theorem 4.2. Let $0<\delta<1$ and let $\alpha \in(0,1)$ be irrational. Then

$$
\operatorname{dim}_{\mathrm{B}} \Omega_{\alpha}^{\delta}\left(D_{0}^{\delta}\right)=\delta \text {. }
$$


Proof. Throughout, we maintain the notation introduced in Section 2.1 when defining the classic Denjoy map. For simplicity of notation, we write $\Omega=\Omega_{\alpha}^{\delta}\left(D_{0}^{\delta}\right)$.

First we show that $\operatorname{dim}_{\mathrm{B}} \Omega \leq \delta$. For $n \in \mathbb{N}$, consider the set

$$
\mathcal{J}_{n}=S^{1} \backslash\left(\bigcup_{-n \leq k \leq n} J_{k}\right) .
$$

It consists of $2 n+1$ disjoint intervals of total length

$$
1-\sum_{-n \leq k \leq n}\left|J_{k}\right|=c_{n} n^{(\delta-1) / \delta},
$$

where the $c_{n}$ are uniformly bounded. Letting $\left\{I_{k}\right\}_{k=1}^{2 n+1}$ denote the $2 n+1$ complementary intervals of $\mathcal{J}_{n}$, we have $\Omega \subset \bigcup_{1 \leq k \leq 2 n+1} I_{k}$. The $\delta+\varepsilon$ covering sum of $\Omega$ can be estimated by covering the union by intervals of the average length, and so by intervals of length $n^{1-1 / \delta} /(2 n+1)$. Using Lemma 4.1, we have

$$
\begin{aligned}
\sum_{k=1}^{2 n+1}\left(\left[\frac{\left|I_{k}\right|(2 n+1)}{\sum_{i=1}^{2 n+1}\left|I_{i}\right|}\right]+1\right)\left(\frac{n^{1-1 / \delta}}{2 n+1}\right)^{\delta+\varepsilon} & \leq \frac{6 n+3}{(2 n+1)^{\delta+\varepsilon}} n^{\delta-1+\varepsilon-\varepsilon / \delta} \\
& =K n^{-\varepsilon / \delta}
\end{aligned}
$$

for a uniformly bounded constant $K$.

As it suffices to consider a geometric sequence of lengths, we have $\operatorname{dim}_{\mathrm{B}} \Omega \leq \delta$.

For the lower bound, let $\left\{U_{k}\right\}$ be a finite cover of $\Omega$ consisting of intervals of uniform length. Without loss of generality, we may assume that there exist $m, n \in \mathbb{N}$ such that no $U_{k}$ intersects $\bigcup_{-m \leq l \leq n} J_{l}$. The orbit segment

$$
\{r \alpha:-m-n-1 \leq r \leq m+n+1\}
$$

contains at least one point in each of the $m+n+1$ contiguous intervals $I_{k}$ of $S^{1} \backslash \bigcup_{-m \leq l \leq n} J_{l}$. This means that the length of any $I_{k}$ is bounded from below by $c_{\delta}(2 n+2 m+3)^{-1 / \delta}$. Moreover, the distance between any two intervals $I_{k}$ and $I_{j}$ with $k \neq j$ is at least $c_{\delta}(\max (n, m))^{-1 / \delta}$.

Thus in order to cover $\Omega$ by intervals of length $c_{\delta}(2 n+2 m+3)^{-1 / \delta}$, we need at least $m+n+1$ intervals. Hence, the covering sum is bounded from below by

$$
0<c_{\delta}^{\delta-\varepsilon} \leq(m+n+1) c_{\delta}^{\delta-\varepsilon}(2 n+2 m+3)^{(\varepsilon-\delta) / \delta} \leq \sum_{k}\left|U_{k}\right|^{\delta-\varepsilon} .
$$

This proves the other direction.

Combining the computation in the second half of the proof of Theorem 4.2 and Lemma 2.3, we obtain: 
Theorem 4.3. Let $0<\delta<1$ and let $\alpha \in(0,1)$ be irrational. Then

$$
\min _{D^{\delta} \in \mathcal{D}^{\delta}} \operatorname{dim}_{\mathrm{LB}} \Omega_{\alpha}^{\delta}\left(D^{\delta}\right) \geq \delta .
$$

Proof. We apply Lemma 2.3 for all $\beta<\delta$.

\section{Proof of Theorem 3.1}

\subsection{Beatty sequences}

Definition 5.1. Let $\alpha \in(0,1)$ be irrational and assume that $\left\{q_{n}\right\}$ is the sequence of denominators for the continued fraction expansion of $\alpha$. For $n_{0} \in \mathbb{N}$, the $n_{0}$-hitting sequence of $\alpha$ is defined to be the subsequence $\left\{r_{k}\right\}_{k \in \mathbb{Z}}$ of integers so that $r_{k} \alpha \in\left[0, q_{n_{0}} \alpha\right]$.

It is a classic result that:

Lemma 5.2 (Hedlund and Morse [5]). Let $\alpha \in(0,1)$ be irrational, let $n_{0} \in \mathbb{N}$ and assume that $\left\{r_{k}\right\}$ is the $n_{0}$-hitting sequence of $\alpha$. Then there exist $\lambda>1$ and $\theta>0$ so that $r_{k}=[k \lambda+\theta]$.

In symbolic dynamics terminology, this means that the sequence $\left\{r_{k}\right\}$ is a Beatty sequence. It is easy to see that a Beatty sequence $\mathcal{B}$ is close to being arithmetic. More precisely, if $r_{k}, r_{k+1} \in \mathcal{B}$ are two consecutive elements in the Beatty sequence defined by the parameters $\lambda>1$ and $\theta>0$, then $r_{k+1}-r_{k}$ takes on only one of two values, either $[\lambda]$ or $[\lambda]+1$.

Maintaining notation as before, we write

$$
\Omega_{\alpha}^{\delta}\left(D^{\delta}\right)=S^{1} \backslash \bigcup_{n \in \mathbb{Z}} J_{n}
$$

The semi-conjugacy $h$ given by Poincaré's Theorem is two-to-one at the endpoints of the intervals $J_{n}$. We write $g_{l}(n \alpha)$ and $g_{r}(n \alpha)$ for the left and right preimages under $h$ on the orbit of $\alpha$, i.e. if $h(x)=h(y)=n \alpha$ and $x, y$ are the standard representatives of the equivalence classes $(x),(y) \in \mathbb{R} / \mathbb{Z}$ with $x<y$ then $g_{l}(n \alpha)=x$ and $g_{r}(n \alpha)=y$. Formally, we should write the equivalence class instead of a representative of this class, but for simplicity we avoid this notation.

5.2. Lower bound for the Hausdorff dimension. We now prove the lower bound in Theorem 3.1.

Proof (first part of Theorem 3.1). Let $\mathcal{J}=\left\{I_{k}\right\}$ be a covering of $\Omega=$ $\Omega_{\alpha}^{\delta}\left(D^{\delta}\right)$ by intervals and choose $t \in \mathbb{R}$ so that the covering sum

$$
\mathfrak{S}^{t}(\mathcal{J})=\sum_{k}\left|I_{k}\right|^{t}>1
$$

Without loss of generality, we may assume that each interval $I_{k}$ has the form $I_{k}=\left[g_{r}(n \alpha), g_{l}(m \alpha)\right]$, where $n=n(k), m=m(k) \in \mathbb{Z}$ and $n<m$. (If not, 
the boundary points of the interval $I_{k}$ fall into an interval $J_{l} \in \mathcal{D}^{\delta}$ and so we could shrink the interval while still covering $\Omega$ with a smaller covering sum.)

Given such a covering of $\Omega$, its image under $h$ covers $S^{1}$. Thus we estimate the distortion of the intervals in the covering of $\Omega$ with respect to the map $h$. If the distortion is not too large, the Hausdorff dimension of the cover cannot be too small, giving a lower bound on the dimension. The asymptotic distortion of a point by taking intervals containing the point and shrinking to it, is nothing but the local Hölder exponent of the map $h$.

Therefore, we need a lower bound for the number

$$
\frac{\log |(n \alpha, m \alpha)|}{\log \left|\left(g_{r}(n \alpha), g_{l}(m \alpha)\right)\right|} \text {. }
$$

In order to estimate the distances in (8), we use (1). To bound the first term on the right hand side of (1), we use the leading term in the sum and so need

$$
\varrho=\inf \{|r| \in \mathbb{N}: r \alpha \in[n \alpha, m \alpha]\} .
$$

We note that $r \alpha \in[n \alpha, m \alpha]$ if and only if $(r-n) \alpha \in[0,(m-n) \alpha]$. Let $n_{0} \in \mathbb{N}$ be the smallest natural number such that $q_{n_{0}} \alpha \in[0,(m-n) \alpha]$. Let $p \in \mathbb{N}$ be the largest number so that $p\left\|q_{n_{0}} \alpha\right\| \leq\|(m-n) \alpha\|$. We note that both $p$ and $q_{n_{0}}$ depend on the difference $n-m$ rather than on $n$. Thus for $0 \leq s \leq p-1$ all the intervals $\left[s q_{n_{0}} \alpha,(s+1) q_{n_{0}} \alpha\right]$ are contained in the interval $[0,(m-n) \alpha]$. Using the fact that $\left[0, q_{n_{0}-2} \alpha\right] \supset[0,(m-n) \alpha]$ and applying (6) twice, we have, for large $n_{0}$,

$$
1 \leq p \leq \frac{\left|\left[0, q_{n_{0}-2} \alpha\right]\right|}{\left|\left[0, q_{n_{0}} \alpha\right]\right|} \leq \frac{q_{n_{0}+1}-q_{n_{0}-1}+2 q_{n_{0}}}{q_{n_{0}-1}-q_{n_{0}-3}} \leq 2 \frac{q_{n_{0}+1}}{q_{n_{0}-1}} .
$$

By Lemma 5.2 the $n_{0}$-hitting sequence of $\alpha$ is of the form $r_{k}=[k \theta+\gamma]$ for some $\theta>1$ and $\gamma>0$. For this sequence, $r_{0}=q_{n_{0}}$ and $r_{1}=q_{n_{0}+1}+q_{n_{0}}$. Indeed, to find the value of $r_{1}$ we note first that $\left(q_{n_{0}+1}+q_{n_{0}}\right) \alpha \in\left[0, q_{n_{0}} \alpha\right]$, as $\left\|q_{n_{0}+1} \alpha\right\| \leq\left\|q_{n_{0}} \alpha\right\|$ and this is the first such occurrence. On the other hand, any point $m \alpha \in\left[0, q_{n_{0}} \alpha\right]$ satisfies $m-q_{n_{0}}>q_{n_{0}+1}$.

More generally, since a Beatty sequence is close to being arithmetic, we see that, for $k>0$,

$$
q_{n_{0}}+k\left(q_{n_{0}+1}-1\right)<r_{k}<q_{n_{0}}+k\left(q_{n_{0}+1}+1\right)
$$

and a similar equation holds for $k<0$. Furthermore, it follows that hitting sequences are defined for more general intervals. Namely, each interval $\left[s q_{n_{0}} \alpha,(s+1) q_{n_{0}} \alpha\right]$ has the hitting sequence $r_{k}+s q_{n_{0}}$. Thus the hitting set for the interval $\left[0, p\left\|q_{n_{0}}\right\|\right] \subset[0,(n-m) \alpha]$ is the set

$$
\bigcup_{k} \bigcup_{0 \leq s \leq p}\left\{r_{k}+s q_{n_{0}}\right\}
$$


and for the interval $\left[0,(p+1)\left\|q_{n_{0}}\right\|\right] \supset[0,(n-m) \alpha]$, the hitting sequence is the set

$$
\bigcup_{k} \bigcup_{0 \leq s \leq p+1}\left\{r_{k}+s q_{n_{0}}\right\}
$$

Write $n=r_{k}+b$ for suitable $k$ and some $b$ with $0 \leq b \leq q_{n_{0}+1}$. Using the above description of the hitting sets for the intervals $\left[0, p\left\|q_{n_{0}}\right\|\right]$ and $\left[0,(p+1)\left\|q_{n_{0}}\right\|\right]$, we estimate $\varrho$ so that

$$
\min _{0 \leq s \leq p}\left(\left|b-s q_{n_{0}}\right|,\left|b-r_{k+1}\right|\right) \leq \varrho \leq \min _{0 \leq s \leq p+1}\left(\left|b-s q_{n_{0}}\right|,\left|b-r_{k+1}\right|\right) .
$$

To do so, we define

$$
\varrho(n, p)=\min _{0 \leq s \leq p+1}\left\{r-n \in\left\{r_{k}+s q_{n_{0}}\right\}_{k \in \mathbb{Z}}\right\} .
$$

We note that $p$ actually depends on $n-m$, and so $\varrho(n, p)$ is just a function of $n$ and $m$.

By (1), the quantity in (8) is greater than or equal to

$$
\frac{\log \|(m-n) \alpha\|}{\log \varrho^{-1 / \delta}}=\delta \frac{\log \|(m-n) \alpha\|}{\log \varrho^{-1}} .
$$

Thus a lower bound for (10) is obtained at the same value which maximizes the function

$$
f(n, p)=\|(m-n) \alpha\| \varrho(n, p) .
$$

If $n_{0}$ is large enough and so $(n \alpha, m \alpha)$ is small, the definition of $p$ implies that

$$
\|(m-n) \alpha\| \leq\left\|(p+1) q_{n_{0}} \alpha\right\|=(p+1)\left\|q_{n_{0}} \alpha\right\| \leq(p+1)\left(q_{n_{0}+1}-q_{n_{0}-1}\right)^{-1}
$$

and

$$
\|(m-n) \alpha\| \geq\left\|p q_{n_{0}} \alpha\right\|=p\left\|q_{n_{0}} \alpha\right\| \geq p\left(q_{n_{0}+1}-q_{n_{0}-1}+2 q_{n_{0}}\right)^{-1}
$$

by using (6) and (7). Moreover,

$$
\begin{aligned}
{\left[\left(q_{n_{0}+1}-1-(p+1) q_{n_{0}}\right) / 2\right] } & \leq \max _{n} \varrho(n, p) \\
& \leq\left[\left(q_{n_{0}+1}+1-(p+1) q_{n_{0}}\right) / 2\right]
\end{aligned}
$$

For $n_{0}$ sufficiently large, the maximum of $f(n, p)$ is attained at some

$$
p_{0} \in\left[q_{n_{0}+1} /\left(4 q_{n_{0}}\right), 3 q_{n_{0}+1} /\left(4 q_{n_{0}}\right)\right],
$$

and so $p_{0} \leq 2 q_{n_{0}+1} / q_{n_{0}-1}$. Combining these estimates we have

$$
\|(m-n) \alpha\| \leq\left(\frac{3 q_{n_{0}+1}}{4 q_{n_{0}}}+1\right)\left(q_{n_{0}+1}-q_{n_{0}-1}\right)^{-1}=C q_{n_{0}}^{-1}
$$

for some constant $C$. Thus by (11) there exist constants $c_{1}>c_{2}$ so that

$$
c_{1} q_{n_{0}+1} \geq \varrho\left(n, p_{0}\right) \geq c_{2} q_{n_{0}+1} \text {. }
$$


This implies that

$$
\begin{aligned}
\frac{\log \|n \alpha-m \alpha\|}{\log \left\|g_{r}(n \alpha)-g_{l}(m \alpha)\right\|} & \geq \frac{\log \|(n-m) \alpha\|}{\log \varrho\left(n, p_{0}\right)^{-1 / \delta}} \\
& \geq \frac{\log \left(C q_{n_{0}}^{-1}\right)}{\log \left(c_{1} q_{n_{0}+1}\right)^{-1 / \delta}}=\delta \frac{\log C^{-1} q_{n_{0}}}{\log c_{1} q_{n_{0}+1}}
\end{aligned}
$$

This shows that for any $\varepsilon>0$ there exists a constant $C(\varepsilon)$ so that

$$
\left|h\left(I_{k}\right)\right|<C(\varepsilon)\left|I_{k}\right|^{\kappa}
$$

where

$$
\kappa=\delta \varliminf_{n \rightarrow \infty} \frac{\log q_{n_{0}}}{\log q_{n_{0}+1}}-\varepsilon .
$$

Since J covers $\Omega_{\alpha}^{\delta}$, the collection $\left\{h\left(I_{k}\right)\right\}_{k}$ covers $S^{1}$. Hence

$$
1=\operatorname{dim}_{\mathrm{H}} S^{1} \leq\left(\delta \varliminf_{n \rightarrow \infty} \frac{\log q_{n_{0}}}{\log q_{n_{0}+1}}\right)^{-1} \operatorname{dim}_{\mathrm{H}} \Omega .
$$

Hence we have the lower bound for the Hausdorff dimension of the minimal set.

Let $\lambda$ denote Lebesgue measure. With a slight modification, the same proof shows:

Corollary 5.3. Let $0<\delta<1$. Assume that $\left\{q_{n}\right\}$ are the denominators in the continued fraction expansion of an irrational $\alpha \in(0,1)$. If $\lambda \circ h$ is the (unique) invariant measure on $\Omega_{\alpha}^{\delta}\left(D^{\delta}\right)$, then

$$
\operatorname{dim}_{\mathrm{H}} \lambda \circ h \geq \delta \varliminf_{n \rightarrow \infty} \frac{\log q_{n}}{\log q_{n+1}} .
$$

Proof. The only change needed is that in (12), we replace $1=\operatorname{dim}_{\mathrm{H}} S^{1}$ by $1=\operatorname{dim}_{\mathrm{H}} \lambda$.

5.3. An upper bound for the dimension of the classic Denjoy map. We now prove that the bounds in Theorem 1.1 are sharp. For the lower bounds in the proof of Theorem 1.1, we used distortion estimates on the semiconjugating map. We showed that for $x$ in a $C / q_{m}$ neighborhood of $c q_{m+1} \alpha$, the distortion is maximized. Unfortunately, this distortion estimate does not suffice for computing an upper bound for the Hausdorff dimension because these intervals only cover a set of full measure and not the whole circle. (See also Section 5.4 for an application.)

To find an upper bound for the Hausdorff dimension, we also need to consider points with less distortion and estimate the dimension for such points. 
Proof (second part of Theorem 1.1). If $\alpha \in \operatorname{Dioph}(1)$, then by the previous sections

$$
\delta=\operatorname{dim}_{\mathrm{B}} \Omega_{\alpha}^{\delta}\left(\mathcal{D}_{0}^{\delta}\right) \geq \operatorname{dim}_{\mathrm{H}} \Omega_{\alpha}^{\delta}\left(\mathcal{D}_{0}^{\delta}\right) \geq \delta,
$$

and so it suffices to consider $\alpha \in \operatorname{Dioph}(\nu)$ with $\nu>1$.

Let $\left\{q_{n}\right\}$ denote the sequence of denominators in the continued fraction expansion of $\alpha \in \operatorname{Dioph}(\nu)$. We have

$$
q_{n+1}-q_{n-1} \geq q_{n+1}-q_{n}
$$

and by passing to a subsequence $\left\{n_{k}\right\}$, since $\nu>1$ for $\varepsilon>0$ such that $1 /(\nu-\varepsilon)<1$, we can assume that

$$
q_{n_{k}+1}-q_{n_{k}-1} \geq q_{n_{k}+1}\left(1-q_{n_{k}}^{1-\nu+\varepsilon}\right) .
$$

By passing to a further subsequence if necessary, we can assume that

$$
q_{n_{k}}^{1-\nu+\varepsilon}<1 / k
$$

The proof is divided into two parts. First we estimate the Hausdorff dimension of level sets with a fixed small Hölder exponent and then we estimate the dimension of the remaining set.

Fix a number $s$ with $1 /(\nu-\varepsilon)<s<1$. Consider the positive integers

$$
s_{m}^{(k)} \in\left(\varepsilon_{k} q_{n_{k}+1},\left(1-\varepsilon_{k}\right) q_{n_{k}+1}\right) \cap \mathbb{N},
$$

where $\varepsilon_{k}=q_{n_{k}}^{(s-1) / s}$. Define intervals

$$
\begin{aligned}
I_{s_{m}^{(k)}} & =\left(s_{m}^{(k)} \alpha-1 / q_{n_{k}}, s_{m}^{(k)} \alpha+1 / q_{n_{k}}\right), \\
\widehat{I}_{s_{m}^{(k)}} & =\left(s_{m}^{(k)} \alpha-1 /\left(2 q_{n_{k}}\right), s_{m}^{(k)} \alpha+1 /\left(2 q_{n_{k}}\right)\right) .
\end{aligned}
$$

Since $s>1 /(\nu-\varepsilon)$, we find that

$$
\lim _{k \rightarrow \infty} \frac{\varepsilon_{k} q_{n_{k}+1}}{q_{n_{k}}}=\infty
$$

By similar calculations to those in Section 5.2, since

$$
\min \left\{|r| \in \mathbb{N}: r \alpha \in I_{s_{m}^{(k)}}\right\} \geq \varepsilon_{k} q_{n_{k}+1},
$$

each of the intervals $I_{s_{m}^{(k)}}$ has a preimage $g\left(I_{s_{m}^{(k)}}\right)$ with length bounded above by

$$
\left|g\left(I_{s_{m}^{(k)}}\right)\right| \leq\left(\varepsilon_{k} q_{n_{k}+1}\right)^{-1 / \delta}
$$

Let

$$
F_{s}=\bigcap_{K \in \mathbb{N}} \bigcup_{k \geq K} \widehat{I}_{s_{m}^{(k)}}
$$

This set represents those points whose distortion is not too small and includes points where the distortion is not maximized.

For $x \in F_{s}$ there exist infinitely many intervals $I_{s_{m}^{(k)}}$ containing $x$ with the distance of $x$ to the endpoints larger than $\frac{1}{4}\left|I_{s_{m}^{(k)}}\right|$. By the Besicovitch 
covering lemma, there is a subcover of $F_{s}$ of multiplicity at most 3 consisting of intervals $I_{s_{m}^{(k)}}$. By (15) we can estimate the distortion:

$$
\begin{aligned}
\varliminf_{k \rightarrow \infty} \frac{\log \left|I_{s_{m}(k)}\right|}{\log \left|g\left(I_{s_{m}^{(k)}}\right)\right|} & \geq \varliminf_{k \rightarrow \infty} \frac{\log \left(2 / q_{n_{k}}\right)}{\log \left(\varepsilon_{k} q_{n_{k}+1}\right)^{-1 / \delta}} \\
& =\varliminf_{k \rightarrow \infty} \frac{\delta \log q_{n_{k}}}{((s-1) / s) \log q_{n_{k}}+\log q_{n_{k}+1}} \\
& =\varliminf_{k \rightarrow \infty} \frac{\delta \log q_{n_{k}}}{\log q_{n_{k}+1}}\left(\frac{1}{\nu} \cdot \frac{s-1}{s}+1\right)^{-1} \\
& =\frac{s \delta}{s-1+s \nu} .
\end{aligned}
$$

Next we estimate the dimension of the complement $S^{1} \backslash F_{s}$. The intervals $I_{s_{m}^{(k)}}$ cover a part of the circle avoiding the points $0, \alpha, \ldots, q_{n_{k}} \alpha$. For $l, r \in$ $\left[0, q_{n_{k}}\right), d(l \alpha, r \alpha) \sim 1 / q_{n_{k}}$, where by $\sim$ we mean that the ratio of the two quantities is bounded from above and from zero uniformly in all parameters. Moreover, $d\left(q_{n_{k}} \alpha, 0\right) \sim 1 / q_{n_{k}+1}$.

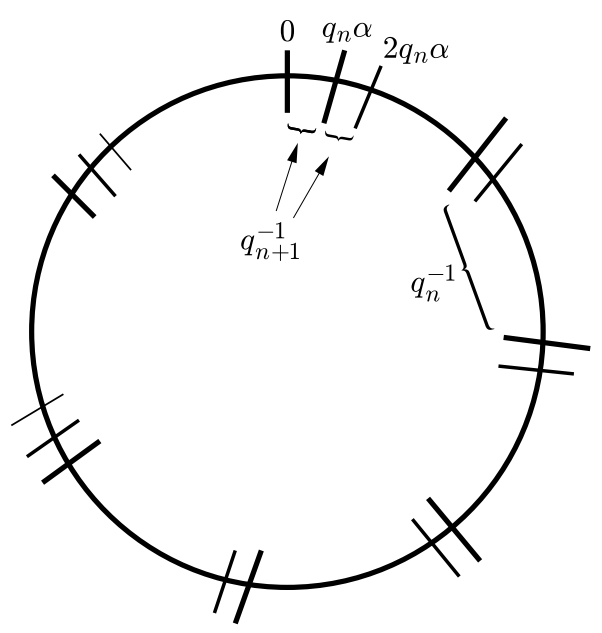

Fig. 1

The gaps between these points are filled up from the left (or from the right, depending on which side of 0 the point $q_{n_{k}} \alpha$ lies) by points at distance $\sim 1 / q_{n_{k}+1}$ for $l \in\left[q_{n_{k}}, q_{n_{k}+1}\right)$. For $0 \leq l<q_{n_{k}}$, each point $l \alpha$ has a new (not necessarily immediate) "neighbor" at the $q_{n_{k}}$ th step, until the gaps are filled. This filling process stops before the last $\varepsilon_{k} q_{n_{k}+1}$ iterates, omitting the $q_{n_{k}}$ gaps which are filled by the remaining iterates with frequencies differing by at most one. This means that the set $\bigcup I_{s_{m}^{(k)}}$ omits $q_{n_{k}}$ gaps of length at 
most

$$
\left(\left[\frac{\varepsilon_{k} q_{n_{k}+1}}{q_{n_{k}}}\right]+1\right) \frac{1}{q_{n_{k}+1}} \leq \frac{2 \varepsilon_{k}}{q_{n_{k}}} .
$$

Hence, the upper box dimension of $S^{1} \backslash F_{s}$ is bounded by

$$
\begin{aligned}
\operatorname{dim}_{\mathrm{B}}\left(S^{1} \backslash F_{s}\right) & \leq \lim _{k \rightarrow \infty}\left(-\frac{\log q_{n_{k}}}{\log \left(2 \varepsilon_{k} / q_{n_{k}}\right)}\right) \\
& =\lim _{k \rightarrow \infty}\left(-\frac{\log q_{n_{k}}}{\log \left(2 q_{n_{k}}^{(s-1) / s} / q_{n_{k}}\right)}\right)=s .
\end{aligned}
$$

Therefore for $\tau$ sufficiently small, $\operatorname{dim}_{\mathrm{B}}\left(F_{s} \backslash F_{s+\tau}\right) \leq s$. This and (16) imply

$$
\operatorname{dim}_{\mathrm{B}} g\left(F_{s} \backslash F_{s+\tau}\right) \leq s \cdot \frac{s \delta}{s-1+s \nu} \leq \frac{\delta}{\nu} \text {. }
$$

We remark that this estimate holds for all $1 /(\nu-\varepsilon)<s<1$ and by the choice of sufficiently small $\varepsilon$, it holds for all $1 / \nu \leq s<1$. Since $\operatorname{dim}_{\mathrm{H}} F_{1}=\delta / \nu$ by Section 5.2 it suffices to show that $\operatorname{dim}_{\mathrm{B}} g\left(S^{1} \backslash F_{1 / \nu}\right) \leq \delta / \nu$. But we already know from $(17)$ that $\operatorname{dim}_{\mathrm{B}}\left(S^{1} \backslash F_{1 / \nu}\right) \leq 1 / \nu$.

By Minkowski's Theorem ([2, p. 48]), the intervals

$$
\left\{\left(n \alpha,\left(n+q_{m} / 4\right) \alpha\right): 0 \leq n \leq q_{m}, m \in \mathbb{N}\right\}
$$

cover all points in the circle that are not linearly independent of $\alpha$ and 1 over the rationals. Using the intervals $\left\{\left(n \alpha,\left(n+q_{m}\right) \alpha\right): 0 \leq n \leq q_{m}, m \in \mathbb{N}\right\}$ in the distortion estimates, we have bounds on the distortion

$$
\varliminf_{n \rightarrow \infty} \frac{\log q_{n+1}^{-1}}{\log \left(n+q_{n+1}+q_{n}\right)^{-1 / \delta}}=\delta .
$$

Therefore the dimension drops by a factor of at least $\delta$ and this implies that $\operatorname{dim}_{\mathrm{B}} g\left(S^{1} \backslash F_{1 / \nu}\right) \leq \delta / \nu$.

Corollary 5.4. Let $0<\delta<1$ and let $\alpha \in \operatorname{Dioph}(\nu)$. Assume that $h$ is the semi-conjugacy given by Poincaré's Theorem for a $C^{1+\delta}$ Denjoy map with rotation number $\alpha$. Then

$$
\operatorname{dim}_{\mathrm{H}} \lambda \circ h=\delta / \nu
$$

Proof. The lower bound is contained in Corollary 5.3. The measure cannot have larger dimension than its support and so we have equality.

5.4. An application to discrepancy. For a sequence $\left\{x_{1}, \ldots, x_{N}\right\}$ of $N$ numbers in $S^{1}$ and a subset $Y \subset S^{1}$, let

$$
A(Y ; N)=\sum_{n=1}^{N} \chi_{Y}\left(x_{n}\right)
$$

count the number of $n$ with $x_{n} \in Y$. 
Definition 5.5. The discrepancy $D_{N}$ of the $N$ numbers $\left\{x_{1}, \ldots, x_{N}\right\}$ in $S^{1}$ is defined to be

$$
D_{N}=\sup _{J}\left|\frac{A(J ; N)}{N}-\right| J||
$$

where the supremum is taken over all subintervals of $S^{1}$.

The discrepancy arises in many situations as an error estimate. Kuipers and Niederreiter [10] prove the following theorem, attributed to Behnke. (This is an exact bound on the discrepancy: the upper estimate is attributed to Hecke and Ostrowski.)

Theorem 5.6 (Behnke [1]). If $\alpha \in(0,1)$ is irrational of Diophantine class $\nu$, then the discrepancy $D_{N}$ of the fractional parts of the sequence $\{\alpha, 2 \alpha, \ldots\}$ satisfies

$$
D_{N} \geq c N^{-1 / \nu+\tau}
$$

for every $\tau>0$ and some constant $c>0$.

Proof. We maintain all the same notation as in Section 5.3.

Since the discrepancy $D_{N}$ is always at least $1 / N$, it suffices to consider $\nu>1$.

We show that for sufficiently large $N$, the estimate in (19) holds with an arbitrary constant $c$. Accounting for the initial portion of the sequence $D_{N}$, we then obtain the estimate of (19) for some $c>0$.

We proceed by contradiction. Assume instead that for some $\tau>0$ and some $c>0$, we have

$$
D_{N} \leq c N^{-1 / \nu+\tau} \text {. }
$$

Assume $\varepsilon /(\nu-\varepsilon)<\tau \nu$. We proceed using a similar method to that used in the proof of Theorem 1.1. Choose the sequence $\left\{q_{n_{k}}\right\}$ (as in the proof of the second part of Theorem 1.1) so that $q_{n_{k}} \leq q_{n_{k}+1}^{1 /(\nu-\varepsilon)}$.

Consider the positive integers

$$
s_{m}^{(k)} \in\left(\frac{1}{4} q_{n_{k}+1}, \frac{3}{4} q_{n_{k}+1}\right) \cap \mathbb{N} .
$$

Define intervals

$$
I_{s_{m}^{(k)}}=\left(s_{m}^{(k)} \alpha-1 /\left(2 q_{n_{k}}\right), s_{m}^{(k)} \alpha+1 /\left(2 q_{n_{k}}\right)\right) .
$$

By similar calculations to those in Section 5.2, we find that for some $c_{3}>0$ independent of $k$,

$$
c_{3} q_{n_{k}+1} \geq \min \left\{|r| \in \mathbb{N}: r \alpha \in I_{s_{m}^{(k)}}\right\} \geq c_{4} q_{n_{k}+1} .
$$

Therefore each of the intervals $I_{s_{m}^{(k)}}$ has a preimage $g\left(I_{s_{m}^{(k)}}\right)$ with length bounded by

$$
\left(\frac{1}{c_{3} q_{n_{k}+1}}\right)^{-1 / \delta} \leq\left|g\left(I_{s_{m}^{(k)}}\right)\right| \leq\left(\frac{1}{c_{4} q_{n_{k}+1}}\right)^{-1 / \delta} .
$$


Let

$$
F_{s}^{(k)}=\bigcup_{m} I_{s_{m}^{(k)}} .
$$

We claim that (20) implies that for sufficiently large $k$, the set $F_{s}^{(k)}$ covers the whole circle and hence $g\left(F_{s}^{(k)}\right) \supset \Omega$.

Indeed, (20) implies that for any interval $J$ of length $1 / q_{n_{k}}$

$$
\begin{aligned}
A\left(J, \frac{1}{2} q_{n_{k}+1}\right) & \geq \frac{q_{n_{k}+1}}{2 q_{n_{k}}}-c 2^{1 / \nu+\tau-1} q_{n_{k}+1}^{1-1 / \nu+\tau} \\
& \geq \frac{1}{2} q_{n_{k}+1}^{1-1 /(\nu-\varepsilon)}-c 2^{1 / \nu+\tau-1} q_{n_{k}+1}^{1-1 / \nu+\tau} \geq \widetilde{c} q_{n_{k}+1}^{1-1 /(\nu-\varepsilon)}
\end{aligned}
$$

for some constant $\widetilde{c}>0$. Thus for sufficiently large $k$, the sequence $\{n \alpha\}_{0 \leq n \leq(1 / 2) q_{n_{k}+1}}$ hits any interval of length $1 / q_{n_{k}}$ and so does the sequence $\left\{(1 / 2) q_{n_{k}+1} \alpha+n \alpha\right\}_{0 \leq n \leq(1 / 2) q_{n_{k}+1}}$. This proves the claim.

Now we estimate the distortion by using (22):

$$
\varliminf_{k \rightarrow \infty} \frac{\log \left|I_{s_{m}^{(k)}}\right|}{\log \left|g\left(I_{s_{m}^{(k)}}\right)\right|}=\varliminf_{k \rightarrow \infty} \frac{-\log q_{n_{k}}}{\log q_{n_{k}+1}^{-1 / \delta}}=\frac{\delta}{\nu} .
$$

Since by $(22)$ the intervals $g\left(I_{s_{m}(k)}\right)$ have approximately the same length for fixed $k$, the above distortion estimate implies that using the coverings $\left\{g\left(I_{s_{m}^{(k)}}\right)\right\}$ we have

$$
1=\operatorname{dim}_{\mathrm{B}} S^{1} \geq \frac{\nu}{\delta} \operatorname{dim}_{\mathrm{B}} \Omega
$$

a contradiction with Theorem 4.2 for $\nu>1$.

\section{References}

[1] H. Behnke, Zur Theorie der diophantischen Approximationen, I, Abh. Math. Sem. Hamburg 3 (1924), 261-318; II, ibid. 4 (1926), 33-46.

[2] J. W. S. Cassels, An Introduction to Diophantine Approximation, Cambridge Univ. Press, Cambridge, 1957.

[3] A. Denjoy, Sur les courbes définies par les équations différentielles à la surface du tore, J. Math. Pures Appl. 2 (1932), 333-375.

[4] K. Falconer, The Geometry of Fractal Sets, Cambridge Univ. Press, Cambridge, 1985.

[5] G. A. Hedlund and M. Morse, Symbolic dynamics II: Sturmian trajectories, Amer. J. Math. 62 (1940), 1-42.

[6] M. R. Herman, Sur la conjugaison différentiable des difféomorphismes du cercle à des rotations, Publ. Inst. Math. Hautes Études Sci. 49 (1979), 5-234.

[7] A. Katok and B. Hasselblatt, Introduction to the Modern Theory of Dynamical Systems, Cambridge Univ. Press, Cambridge, 1995.

[8] Y. Katznelson and D. Ornstein, The differentiability of the conjugation of certain diffeomorphisms of the circle, Ergodic Theory Dynam. Systems 9 (1989), 643-680. 
[9] Y. Katznelson and D. Ornstein, private communication, 1999.

[10] L. Kuipers and H. Niederreiter, Uniform Distribution of Sequences, Wiley, New York, 1974.

[11] A. Norton, Denjoy's Theorem with exponents, Proc. Amer. Math. Soc. 127 (1999), 3111-3118.

[12] A. Pinto, Poster session, Edinburgh and private communication, 2000.

[13] H. Poincaré, Mémoire sur les courbes définies par une équation différentielle, I, J. Math. Pures Appl. (3) 7 (1881), 375-422; II, 8 (1882), 251-286; III , (4) 1 (1885), 167-244; IV, 2 (1886), 151-217.

[14] J.-C. Yoccoz, Conjugaison différentiable des difféomorphismes du cercle dont le nombre de rotation vérifie une condition diophantienne, Ann. Sci. École Norm. Sup. 17 (1984), 333-361.

Department of Mathematics

The Pennsylvania State University

University Park, PA 16802, U.S.A.

E-mail: kra@math.psu.edu
Centre for Mathematical Sciences

LTH, Lund University

Box 118

SE-221 00 Lund, Sweden E-mail: joerg@maths.lth.se

Received on 18.12.2000

and in revised form on 11.2.2002 